\title{
Hydrodynamic Cell Enrichment in Double Spiral Microfluidic Channels
}

\author{
Jiashu Sun, ${ }^{a}$ Mengmeng Li, ${ }^{a}$ Chao Liu, ${ }^{b}$ Guoqing Hu, ${ }^{b}$ Xingyu Jiang ${ }^{a}$ \\ ${ }^{a}$ CAS Key Lab for Biological Effects of Nanomaterials and Nanosafety, National Center \\ for NanoScience and Technology, Beijing, 100190, China. \\ ${ }^{\mathrm{b}}$ Institute of Mechanics, Chinese Academy of Sciences, 100190, China.
}

\begin{abstract}
Rapid cell separation and enrichment has attracted significant attention because of its important applications in cell biology studies and biomedical diagnostics. we have developed a compact microfluidic platform, composed of 6-loop double spiral microchannels, to separate cancer cells from blood cells. The curved geometry induces a Dean drag force acting on cells to compete with the inertial lift, resulting in different equilibrium positions for different sized cells. The separation chip is characterized with the mixture of 4T1 cells and the murine blood cells at different flow rates, with decent separation and enrichment efficiency.
\end{abstract}

\section{Introduction}

Rapid cell separation and concentration has attracted significant attention because of its important applications in cell biology studies and biomedical diagnostics (1). Traditional techniques used to separate cells by size include centrifuge and microfiltration. Other innovative techniques, e.g., fluorescence-activated cell sorting (FACS), magnetically activated cell separation (MACS), acoustic cell filter, and cell affinity chromatography (CAC), have also been developed as more sophisticated tools to conduct cell separation based on cell properties. Conventional techniques used to separate different size cells include centrifuge and microfiltration, which are bulky, laborious and sometimes perform ineffectively in separating cells over a broad size range $(2,3)$.

Microfluidic methods, taking advantage of unique hydrodynamic effects under small length scales and low Reynolds numbers, allow precise and continuous cell separation in a compact format with throughput comparable to traditional techniques(4-6). A simple method is to construct micropost arrays that are separated from each other by different distances, serving as microsieves to separate different sized cells. Besides, the particular geometries of microfluidic systems will induce additional hydrodynamic forces, which could be adopted to separate cells by size. For example, using inertial lift and Dean drag forces in asymmetric curving channels, the randomly distributed particles of different sizes can be focused into several steams that are sizedependent, leading to equilibrium separation at a high flow rate The most remarkable feature of size-based passive microfluidic separation techniques is to eliminate the need of cell labeling, as well as applying any external forces except driving pressures (7). However, most passive-based cell sorters published so far have been focused on proof-ofconcept demonstration by using specified particle mixtures or pre-conditioned biological samples, and hence lacking the biological sense. 


\section{Theory}

Many factors, including geometries of channel, flow conditions, and properties of suspending liquid medium, influence the performance of the microfluidic particle/cell sorting and concentration devices. We developed a double spiral microchannel with 6loop for each direction to particle/cell manipulation, as illustrated in Fig. 1(a). At a relatively high Reynolds number, a secondary cross-section flow (Dean flow) will develop in curved microchannels due to the action of centrifugal forces. Neutrally buoyant particles suspended in liquids are also subjected to drag force and lift force, which result in differential particle migration within the microchannel, as illustrated in Fig. 1(b). The equilibrium positions of particles with different sizes are determined by the competition between hydrodynamic (drag and lift) forces acting on the particles in highvelocity flows. To describe the motion of particles in closed microchannels, two dimensional Reynolds numbers are introduced here: the channel Reynolds number $\left(R e_{c}\right)$ and the particle Reynolds number $\left(R e_{p}\right)$,

$$
\begin{aligned}
& \operatorname{Re}_{C}=\frac{\rho U_{m} D_{h}}{\mu} \\
& \operatorname{Re}_{P}=\operatorname{Re}_{C} \frac{a^{2}}{D_{h}^{2}}=\frac{\rho U_{m} a^{2}}{\mu D_{h}}
\end{aligned}
$$

At small particle Reynolds number $R e_{p}<<1$, the magnitude of the lift forces responsible for movement away from the channel center and walls, can be written as

$$
F_{Z}=\frac{\mu^{2}}{\rho} \operatorname{Re}_{P}^{2} f_{C}\left(\operatorname{Re}_{C}, x_{C}\right)
$$

According Eq. [2], the particle size has a dramatic influence on this lateral force. In a straight channel, the lift coefficient vanishes at the axis of the channel, increases in magnitude with distance towards to the wall until a maximum is reached, and then decreases again. When the Reynolds number is increased, the equilibrium position of a particle in quadratic flow is moved towards the wall. The curvature of the present doublespiral microchannel creates additional transverse drag forces on particles and alters the position of flowing particles.

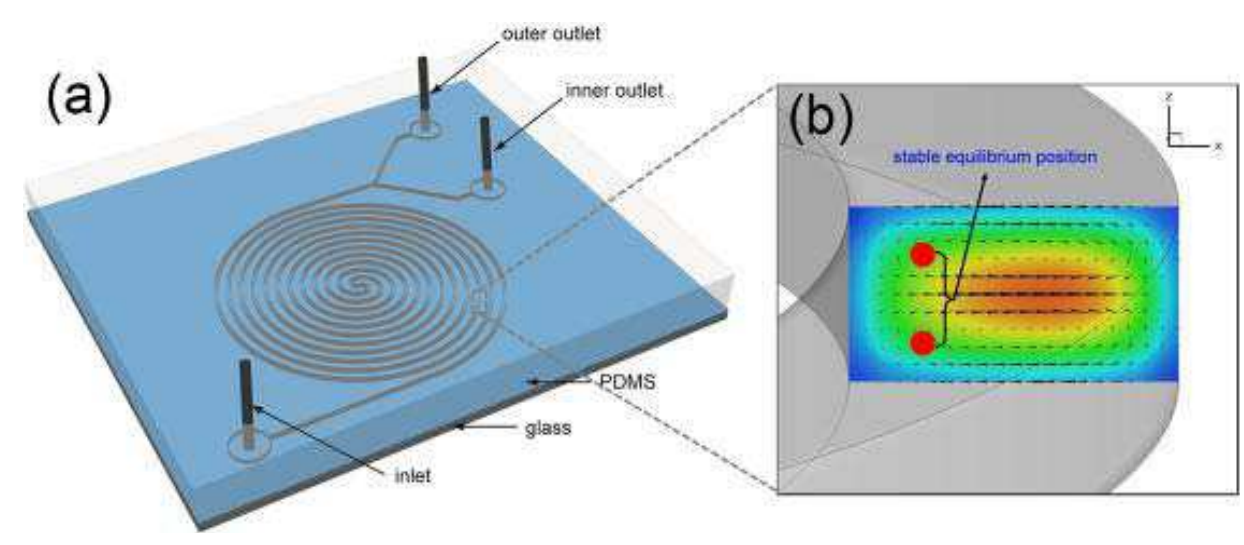

Figure 1. (a) Schematics of the microfluidic cell sorter containing 6-loop double spiral microchannels for particle/cell separation; (b) Illustration of the Dean flow inside a curved microchannel and the stable equilibrium positions of the particles. 


\section{Materials and methods}

The microfluidic cell sorter for particle/cell separation consists of 6-loop double spiral microchannels with one inlet and two symmetric bifurcated outlets, as depicted in Fig. 1. The channel connected with the inlet first spirals in the counterclockwise direction to form 6 loops, changes its direction through a center junction, spirals out by another 6loop clockwise channels, and ends with bifurcated outlets. The spiral microchannels are $150 \mu \mathrm{m}$ wide and $80 \mu \mathrm{m}$ deep, and the distance between two adjunction loops is $450 \mu \mathrm{m}$. The outlet towards to the double spiral channels is termed as the inner outlet, and the other is the outer outlet.

The microfluidic device is fabricated using standard soft-lithography techniques with SU8 master mold on a silicon substrate [8,9]. After the PDMS slab is peeled from the mold, three ports at the inlet and outlets are punched through the PDMS with a flat tip needle. The PDMS slab is then bonded with a glass substrate $(25 \mathrm{~mm} \times 75 \mathrm{~mm})$ post oxygen plasma treatment. Three plastic tubes are inserted through the ports and glued by the liquid PDMS on the top of device. The assembled device is finally placed into an oven at $70{ }^{\circ} \mathrm{C}$ for $30 \mathrm{~min}$ to cure the liquid PDMS and increase bonding.

In the experiment for separating cells, the channels were first filled with PBS, followed by loading the mixture solution of 4T1 cells and murine blood cells via the pump. The assembled device was mounted onto the stage of a Leica DMI 6000 microscope. Fluorescent streak images were obtained by a CCD camera with exposure time of $5 \mathrm{~s}$ and superimposed using Adobe Photoshop CS4.

\section{Results and Discussion}

We applied the microfluidic platform to sort and concentrate 4T1 cells from murine blood cells at a mixture concentration of $2 \times 106 \mathrm{cells} / \mathrm{ml}$ in PBS. The average size of the 4T1 cells is around $15 \mu \mathrm{m}$ in diameter, significantly larger than the surrounding murine blood cells (erythrocytes are $6 \mu \mathrm{m}$ and peripheral blood lymphocytes are 7-10 $\mu \mathrm{m}$ ). Due to this large differences in cell size, we can simply differentiate 4T1 cells from blood cells by analyzing the bright-field microscopic images. The initial concentration ratio between $4 \mathrm{~T} 1$ and blood cells in the cell mixture before separation was $0.86 \%$. After cells traversing through 6-loop double spiral channels at a certain flow rate, most 4T1 cells and partial of blood cells migrated to the inner outlet, and most blood cells and few 4T1 cells moved to the outer outlet, as seen in Fig. 2. By counting more than 1000 cells in the inner and outer outlets, we found that the 4T1 cells in the inner outlet were concentrated by 3.93-fold at the flow rate of $10 \mathrm{~mL} / \mathrm{hr}$, and 5.23-fold at $40 \mathrm{~mL} / \mathrm{hr}$ (Fig. 2). At a throughput of $40 \mathrm{~mL} / \mathrm{hr}$, the normalized $4 \mathrm{~T} 1$ concentration at the outer outlet is 0.165 , which can be read from Figure 6 . Given the initial $4 \mathrm{~T} 1$ ratio that is $0.86 \%$, the ratio of $4 \mathrm{~T} 1$ cells to the collected cell samples at the outer outlet is $0.14 \%$, and the ratio of blood cells equals to $99.86 \%$. Similarly, based on the normalized $4 \mathrm{~T} 1$ concentration ratio of 5.23 , we can obtain that the ratio of $4 \mathrm{~T} 1$ cells at the inner outlet is $4.5 \%$ and that of blood cells is $95.5 \%$. At a throughput of $10 \mathrm{~mL} / \mathrm{hr}$, the normalized $4 \mathrm{~T} 1$ concentration at the outer and the inner outlet is 0.44 and 3.93, respectively, yielding a purity of $4 \mathrm{~T} 1$ cells to be $0.37 \%$ and $3.38 \%$.

Although the concentration ratio of tumor cells in the current design is considerably lower compared to immuno-binding methods or common microfluidic filtration methods, our passive microfluidic technique allows label-free and size-based cell separation with a high throughput. Moreover, simplicity of the system makes it suitable for preliminary 
separation of biological samples that have a size difference. More importantly, our passive cell separation technique allows easy retrieval of target cells that remain viable and intact, which could be used for further cell culture, gene analysis, and cancer prognosis. In order to enhance the separation efficiency and concentration ratio, designing of multiplex cascading of separation circuits or integration of a chip-based detection downstream to refine the collected cells should be attempted in future study.
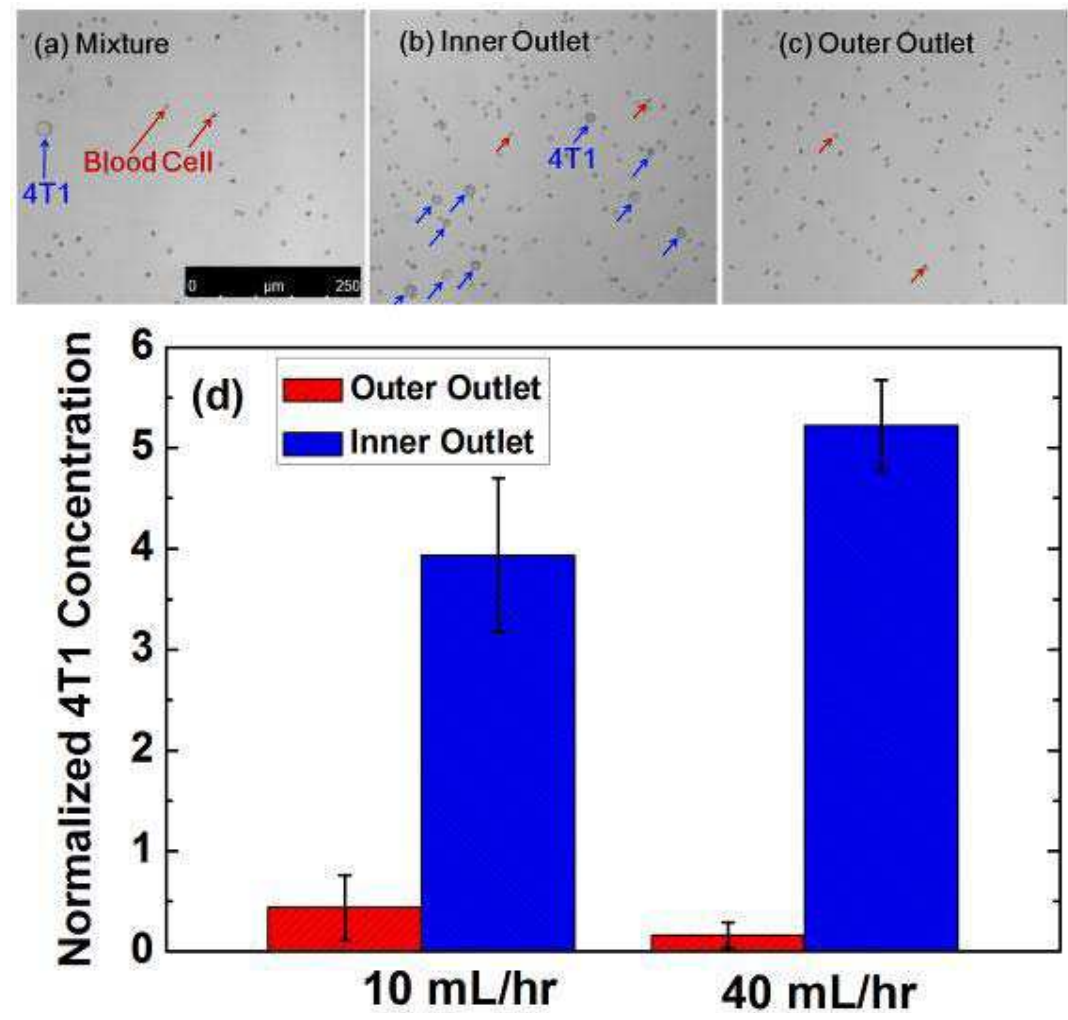

Figure 2. (a-c) Microscopic images for the mixture of 4T1 and blood cells, the inner outlet and the outer outlet at a flow rate of $40 \mathrm{~mL} / \mathrm{hr}$; (d) The normalized 4T1 concentration at the inner and outer outlets at two flow rates.

\section{Acknowledgments}

J.S. and X.J. acknowledge financial support from MOST (2011CB933201, 2009CB930001), NSFC (51105086, 21025520, 90813032), and CAS (KJCX2-YW-M15). G.H. acknowledges financial support from MOST (2011CB707604) and CAS(KJCX2YW-H18).

\section{References}

1. D.R. Gossett, W.M. Weaver, A.J. Mach, S.C. Hur, H.T.K. Tse, W. Lee, H. Amini, D. Di Carlo, Anal. Bioanal. Chem., 397, 3249 (2010).

2. H. Tsutsui, C.M. Ho, Mech. Res. Commun., 36, 92 (2009).

3. P.G. Schiro, M. Zhao, J.S. Kuo, K.M. Koehler, D.E. Sabath, D.T. Chiu, Angew Chem Int Ed Engl, 51, 1 (2012).

4. J. Sun, C.C. Stowers, E.M. Boczko, D. Li, Lab Chip, 10, 2986 (2010). 
5. B. Yuan, Y. Jin, Y. Sun, D. Wang, J.S. Sun, Z. Wang, W. Zhang, X.Y. Jiang, $A d v$ Mater, 24, 890 (2012).

6. K.H. Kang, Y. Kang, X. Xuan, D. Li, Electrophoresis, 27, 694 (2006).

7. W.C. Lee, A.A.S. Bhagat, S. Huang, K.J. Van Vliet, J. Han, C.T. Lim, Lab Chip, 11, 1359 (2011).

8. J.S. Sun, S.K. Vajandar, D.Y. Xu, Y.J. Kang, G.Q. Hu, D.Q. Li, D.Y. Li, Microfluid. Nanofluid., 6, 589 (2009).

9. J. Sun, Y. Gao, R.J. Isaacs, K.C. Boelte, C.P. Lin, E.M. Boczko, D. Li, Anal Chem, 84, 2017 (2012). 